\title{
Recognition by Familiarity Is Preserved In Parkinson's Without Dementia and Lewy-Body Disease
}

\author{
Salvador Algarabel \\ University of Valencia \\ Joaquín Escudero \\ General Hospital, Valencia, Spain \\ Vicente Peset \\ General Hospital, Valencia, Spain \\ Lina-Marcela Cómbita \\ University of Granada
}

\author{
Lucía-Azahara Rodríguez \\ University of Granada \\ Manuel Fuentes \\ University of Valencia \\ Alfonso Pitarque \\ University of Valencia \\ Jose F. Mazón \\ General Hospital, Valencia, Spain
}

\begin{abstract}
Objective: The retrieval deficit hypothesis states that the lack of deficit in recognition often observed in patients with Parkinson's disease is because of the low retrieval requirements of the task, given that these patients have retrieval and not encoding deficits. To test this hypothesis we investigated recognition memory by familiarity in Parkinson's patients and in patients with Lewy Bodies disease and Parkinson with dementia. Method: We analyzed to what extent the experimental groups were able to recognize by familiarity in a typical yes/no recognition memory task. The experimental groups were patients with early nondemented Parkinson's disease, advanced nondemented Parkinson's disease, demented Parkinson's patients, and patients with dementia with Lewy Bodies. We compared their performance with a group of young and another group of old healthy participants. The estimation of familiarity was made by analyzing recognition of word targets and distractors consisting of combinations of different letters in comparison with a condition in which targets and distractors were composed of similar letters, even though subjects were unaware of the independent variable. Results: The results indicate that familiarity was used at the same level by controls, patients with early Parkinson's disease and patients with dementia with Lewy Bodies. Although late Parkinson patients also used familiarity, its effect was only marginally significant. Patients with Parkinson's disease and dementia were not capable of using familiarity in recognition memory. Conclusions: Our results support the retrieval deficit hypothesis as Parkinson's patients without dementia show no deficit in a situation in which the retrieval requirements are minimal.
\end{abstract}

Keywords: familiarity, recognition memory, Parkinson's disease, Parkinson's disease with dementia, Dementia with Lewy Bodies

Behaviorally, Parkinson's disease (PD) is characterized by the presence of resting tremors, rigidity, and bradikinesia, among other general symptoms. Although cognitive deficits did not form part of the initial clinical description of PD, contemporary research has shown that they may play an important role as early predictors of the appearance of dementia (Aarsland, Brannick, Larsen, Tysnes, \& Alves, 2008; Emre, 2003; Verbaan, Marinus, Visser, van Rooden, Stiggelbout, Middelkoop, \& van Hilten, 2007). In general, PD patients show deficits in executive, visuospatial, and

Salvador Algarabel, Manuel Fuentes, and Alfonso Pitarque, Methodology Department, University of Valencia; Lucía-Azahara Rodríguez and Lina-Marcela Cómbita, Experimental Psychology Department, University of Granada; Joaquín Escudero, Vicente Peset, and Jose F. Mazón, Department of Neurology, General Hospital, Valencia, Spain.

This research was supported by Grant SEJ2007-60521 from the Dirección General de Investigación Científica y Técnica (Spanish Ministry of Education and Technology).

Correspondence concerning this article should be addressed to Salvador Algarabel, Facultad de Psicologia, Universidad de Valencia, Blasco Ibanyez, 21, 46010 Valencia, Spain. E-mail: Salvador.Algarabel@uv.es attentional functions, with only quantitative differences existing between those with and without dementia. However, a recent study using a neuropsychological battery to evaluate nonmedicated patients (Aarsland et al., 2008) affirmed that only a small proportion of those with mild cognitive impairment are of the amnestic type The general consensus is that PD patients show mild-to-strong deficits in declarative memory tasks requiring the use of retrieval strategies, such as when free recall is tested, but smaller deficits or none at all in those that do not, such as recognition tasks (Whittington, Podd, \& Kan, 2000). This conclusion may be an oversimplification, at least with respect to the analysis of performance in recognition tasks. Currently, there are a number of theories that attempt to explain how recognition occurs. One-process theories postulate that recognition is achieved by a pure familiarity process in which trace strength is evaluated, whereas dual-process views complement familiarity with a second process of recollection of the study episode. Familiarity and recollection are defined in relation to each other (Yonelinas, 2002); the former is faster than the latter, and each has its own electrophysiological profile and is differentially affected by both brain lesions and experimental dissociations produced by a number of variables (see Yonelinas, 2002, for a review). In lay terms, familiarity is that sense of "déjà 
vu" or fluency experienced upon seeing someone or something that suggests it is not the first time we have the encounter. This experience of familiarity is produced without any other details that allow us to place the event in space and time. This last aspect of the memory episode is a landmark of recollection.

If we take into account the dual view, which is dominant in neuroscience, an experiment should be composed of a number of study-test trials in which participants use a combination of both processes in accordance with the conditions of the task. Therefore, a recognition task may be similar to a free-recall task, and may lead to contradictory conclusions with regard to performance, because of a shifting balance between the use of familiarity and recollection. In general, one would expect to observe deficits in recognition when recollection plays a role, but much less so when the task is more familiarity-based. The root of the problem in analyzing the published data in this field is the assumption often made by neuropsychologists that recognition does not require the use of retrieval mechanisms, in line with the old generationrecognition theory of free recall and recognition (Crowder, 1976).

In a meta-analysis of the literature, Whittington, Podd, and Kan (2000) determined that the size of the recognition memory deficit in Parkinson's patients is moderated mainly by length of illness, medication, and presence of associated dementia. We could safely assume at this point that this deficit in recognition memory is related more to recollection than familiarity. Studies on prospective memory in PD patients (Costa, Peppe, Caltagirone, \& Carlesimo, 2008) support this interpretation. Costa et al. (2008) observed that PD patients were deficient in the retrospective component of a prospective task, usually attributed to problems in recollecting information from memory. Nevertheless, the hypothesis is far from established, and constitutes the main research goal of this article.

Alternatively, one may form an idea of the extent to which familiarity could be deficient in Parkinson's disease by analyzing the research on implicit memory. Implicit memory is characterized by changes in performance and is a product of previous exposure to stimuli without the conscious intervention of the subject. Although familiarity is produced in the context of a task in which the subject makes a conscious effort to retrieve information, the fact that it is associated with a general sense of "déjà vu" without specific references to the past makes both paradigms comparable. In this context, when Parkinson's patients were tested in a serial reaction time paradigm for the implicit learning of key press sequences (Nissen \& Bullemer, 1987), the results were inconsistent (see Siegert, Taylor, Weatherall, \& Abernethy, 2006). A meta-analysis of six such studies (Siegert et al., 2006) suggested the presence of a modest deficit. However, when using verbal materials, normal lexical priming has been obtained only in nondemented patients (Heindel, Salmon, Shults, Walicke, \& Butters, 1989). Finally, the literature concerning artificial grammar learning (Peigneux, Meulemans, Van der Linden, Salmon, \& Petit, 1999; Reber \& Squire, 1999; Witt, Nuhsman, \& Deuschl, 2002) indicates that PD patients are able to experience intact implicit learning even in the advanced stages of the illness and independently of medication, particularly when the task does not require complex associative requirements (Smith \& McDowall, 2006). In this way, the evidence regarding implicit memory and PD, at least when verbal material is used, does suggest the absence of deficits in familiarity in PD patients (see also Gawrys, Jamrozik, Janik, Friedman, \& Kaczmarek, 2008) when they are required to respond in a recognition paradigm.

Finally, as far as we are aware, the only study that has aimed to establish whether recollection and/or familiarity in a recognition paradigm are damaged in Parkinson's disease with no dementia is that by Davidson, Anaki, Saint-Cyr, Chow, and Moscovitch (2006). They concluded that Parkinson's patients use recollection at the same level as controls but use familiarity at a lower level. This conclusion is controversial given that, as previously mentioned, Parkinson's patients show deficits in aspects other than recognition in episodic memory tasks (e.g., Emre, 2003; Whittington, Podd, \& Stewart-Williams, 2006). Anatomically speaking, there is not much evidence in favor of the existence of deficits in familiarity over recollection in PD. Although damages in the basal ganglia take place during the development of Parkinson's disease, there are no data that associate these structures with familiarity, although a connection through higher cortical structures may exist. There is some evidence that nonverbal implicit learning (Siegert et al., 2006) is associated with the integrity of basal ganglia, but the hippocampal and para-hippocampal areas are the structures most likely to be associated with familiarity. Data obtained with mild cognitive impairment and Alzheimer's disease patients support this (Algarabel, Escudero, Mazón, Pitarque, Fuentes, Peset, \& Lacruz, 2009). In fact, some theories relate perirhinal and hippocampal integrity with the intact functioning of familiarity and recollection, structures not involved in the development of Parkinson's disease in the early stages.

Given the previously described inconsistencies and lack of data, we aimed to confirm the possible existence of familiarity deficits in Parkinson's disease with different years of evolution and in patients with dementia with Lewy Bodies (DLB). Years of evolution has been identified as a significant factor in the observation of recognition deficits in Parkinson's (Whittington et al., 2000). Additionally, given that the presence of dementia is another significant factor, we chose to include a group of Parkinson patients with dementia, and an additional group with DLB. The reason for the inclusion of this last group is because of their similarity to those of Parkinson's with dementia (McKeith, 2007; McKeith et al., 2004). The clinical similarities and, consequently, the confusion between Parkinson patients with dementia on the one hand and with DLB on the other arises from the presence of Lewy bodies and dopaminergic deficits in similar brain structures in both cases. Given the degree of similarity between the two clinical conditions, there are currently no clinical criteria separating DLB and PD with or without dementia (McKeith, 2007). Neurologists (McKeith et al., 2004) use the 1-year rule to differentiate between the two diagnoses, so that when dementia appears within the first year of Parkinson diagnosis, patients are categorized as DLB and PDD otherwise.

Although recognition is an experimental paradigm that is less reliant on retrieval strategies than free or cue recall, its use is well documented. A lack of sufficient experimental control may explain the contradictory data reported in the literature with respect to the evaluation of possible performance deficits in Parkinson's patients. To avoid as much as possible the involvement of such strategies, we chose to assess familiarity directly with a paradigm (Algarabel, Pitarque, Tomás, \& Mazón, 2010; Parkin, Ward, Squires, Furbear, Clark, \& Townshend, 2001) that is simple to apply and easily 
comprehended by a patient population with potentially serious cognitive deficits. The Parkin et al.'s paradigm offers a direct estimation of the use of familiarity processes in responding. It consists of presenting a series of words formed by a subset of letters of the alphabet mixed with others formed by a different subset for the recognition test (nonoverlapping condition; NO). The NO condition is compared with an overlapping one, in which all words are formed by letters extracted from both subsets (overlapping condition; O). We selected this task because it reflects the idea of what familiarity is: the "déjà vu" feeling without the ability to identify its source, and in consequence, a product of an automatic retrieval process. A previous study by our group (Algarabel et al., 2010) has revealed that the Spanish version of the task produces stronger perceptual familiarity than the English one, because of the different letter probabilistic structures of the two languages.

In conclusion, based on the previously explained arguments, we expected that, although recognition would vary between groups, and would likely to be poor among DLB and Parkinson patients with dementia, familiarity, given its automatic and low resource requirement nature, would be similar in all patients unaffected by dementia if the retrieval deficit was maintained. This hypothesis (Higginson, Wheelock, Carroll, \& Sigvardt, 2005) establishes that subcortical dementias (PD and Huntington's disease; Cummings \& Benson, 1984) are characterized by retrieval rather than encoding deficits. On the other hand, in accordance with the abovementioned literature, we expected to observe deficits in familiarity in the groups of patients affected by dementia. Finally, we explored familiarity in two groups of PD patients differentiated by the number of years of evolution of their illness. In this way, we aimed to assess familiarity in groups of patients differing in the key variables affecting recognition performance (see Whittington et al., 2000). Of special relevance to any evaluation of the retrieval deficit hypothesis is the performance of patients without dementia, whose familiarity performance would be expected to be intact.

\section{Method}

\section{Participants}

Two groups of idiopathic nondemented PD patients (with 20 and 19 participants, respectively), one group of demented PD patients (10 participants) and one group of DLB patients (17 participants) were recruited from the Department of Neurology of the Hospital General of Valencia, Spain. In addition, 16 healthy elderly and 16 healthy young controls were included in the study. Parkinson's disease patients without dementia were divided according to the number of years since the start of symptoms into either the early $(M=2.93, S D=1.28)$ or advanced $(M=11.53$, $S D=4.94$ ) PD group. In the PD1 group, five participants gave a Hoehn and Yahr (1967) rating of Stage I, and the remaining participants gave a rating of Stage II. In the PD2 group, 17 participants gave a Hoehn and Yahr rating of Stage III, while the remaining subjects gave a rating of Stage IV. All patients in the PDD group gave a rating of Stage III. The groups were matched in education and age (see Table 1), with the exception of the PD patients with dementia, who were slightly older than those in the early PD group.

At the time of being tested, the patients were receiving medication: dopaminergic treatment with carbidopa-levodopa and or
MAO inhibitors (selegiline and rasagiline) and or dopaminergic agonists (rotigotine, pramipexole, ropinirole). Rivastigmine was also prescribed in the case of those with DLB and PD with dementia. All patients were community dwelling, had normal or corrected-to-normal vision and hearing and were tested for cognitive impairment, episodic, semantic and working memory, perceptual and visual-constructive capacity, and executive function. Table 1 presents the demographic data of the study population together with that of the tests used to assess the aforementioned functions.

Diagnosis was carried out after studying the medical history of each patient and carrying out a physical, neurological, neuropsychological, and psychiatric examination. Diagnosis for the PD group was based on the United Kingdom Parkinson's disease Society Brain Bank's Diagnostic Criteria (Hughes et al., 1992). These criteria require the presence of at least two of the following three symptoms: (1) resting tremor, (2) rigidity, and (3) bradykinesia. In addition, (4) a good response to levodopa was a further criterion, while (5) each of the two PD groups were defined by less/more than 6 years of illness development, and (6) a neurological exam had to have been performed within the last year. Exclusion criteria for the PD groups were poor response to anti-Parkisonian medication, significant past episodes of psychiatric or neuropsychological disorder, presence of other causes of dementia or other brain illnesses (vascular injury, surgical brain procedure, craneoencephalic trauma), or a history of alcohol or drug abuse. Diagnosis in the PD group with dementia was according to DSM-IV criteria (American Psychiatric Association, 2000). Diagnosis in the DLB group was based on attention fluctuations, recurrent visual hallucinations and motor features of Parkinsonism, as described in the third report of the DLB consortium (McKeith et al., 2005). Exclusion criteria for DLB were similar to those of Parkinson's, but also included a very serious attention deficit that would make it impossible to perform the experimental test. The elderly control group was comprised of partners of patients referred to the Neurology Unit of the General Hospital of Valencia (Spain) for evaluation who showed no signs of depression, alcoholism, drug intake, dementia, or any other neurological disorder.

\section{Materials and Procedure}

The materials consisted of 124 Spanish words of between three and nine letters in length. In the NO condition, two lists of 25 words each were composed entirely of the following letters of the Spanish alphabet: a, e, u, r, n, d, b, g, z, j, x, k, w (list A), or o, i, $\mathrm{c}, \mathrm{t}, \mathrm{s}, \mathrm{l}, \mathrm{p}, \mathrm{m}, \mathrm{v}, \mathrm{f}, \mathrm{h}, \mathrm{q}, \tilde{\mathrm{n}}$ (list B). The remaining two lists were composed of words containing letters from both sets, with the restriction that each word had to contain at least one letter from each of the two abovementioned pools of letters. The word lists were equated in mean frequency per two million (Alameda y Cuetos, 1995) (means of 45.50, 45.40, 45.28, 45.28, and SDs of 93.56. 67.59, 83.72, 53.03, respectively) and in length (means of $5.25,5.20,5.28$, and 5.24 , and $S D$ s of, $1.04,1.30,1.49,1.27$, respectively). Three additional words with similar characteristics were included at the beginning and another three at the end of each list to mitigate the primacy and recency effects. These words were presented but never tested. In conclusion, four lists of 31 words ( 25 


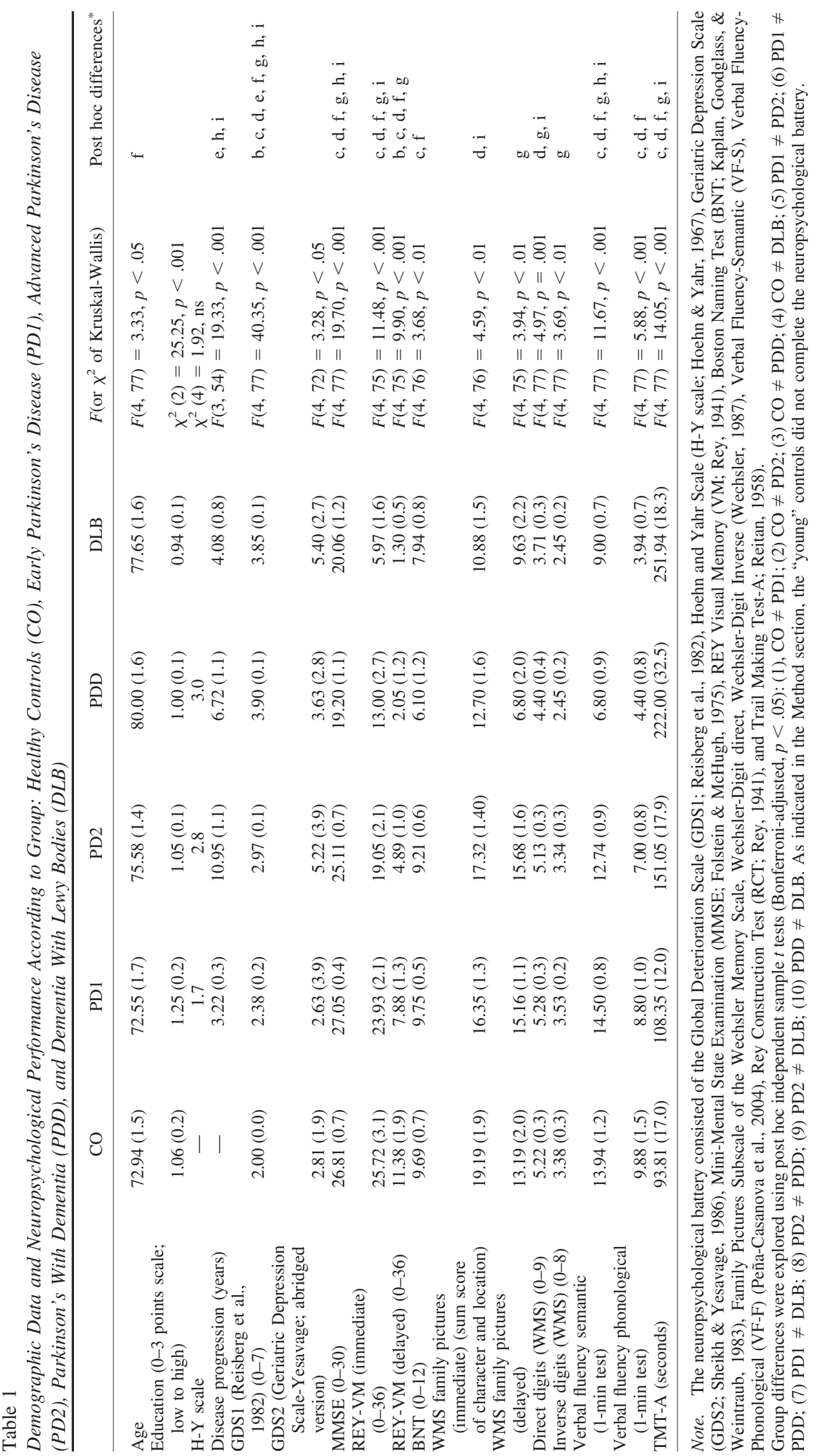


experimental words and six fillers) were established. Two of the lists defined the NO condition, and the remaining two defined the $\mathrm{O}$ condition.

All de groups with the exception of the students underwent the neurosychological examination (individually and in a continuous session). The DLB patients were tested in their homes. The study was approved by the institutional review board of the Valencia General Hospital, and each patient gave his or her written informed consent to be included in the study. The young controls participated in a single experimental session run for two groups of eight participants.

After the neuropsychological examination, patients and elderly controls completed the experimental task, which was presented to them on a laptop computer screen controlled by e-prime software (Schneider, Eschman, \& Zuccolotto, 2002). When necessary, an experimenter keyed in the response verbally expressed by the patient. The task consisted of two study-test lists, one with the NO stimuli and the other with $\mathrm{O}$ stimuli, which were counterbalanced among the participants. The $\mathrm{O}$ list presentation and test was identical to the NO one, except for stimulus composition.

In the NO condition, the stimuli were randomly divided into two lists of 25 words each, which were used as targets and mixed with the remaining 25 distracter words. The target/distracter status was counterbalanced across subjects. Type of list (O-NO) was a within subject variable. At study, 26 words (of which six were fillers) were presented for 2,000 ms each. Following the study phase there was a 5 min interval during which a secondary task was presented (Toulouse \& Pieron, 1986). This task consisted of presenting the subject with a simple geometric drawing. He or she then had to cross out those similar to the sample among 800 drawings on a sheet of paper. This was a paper and pencil task.

The recognition test consisted of the random appearance on the computer screen of 25 studied words (no fillers) plus 25 distracters. The participant made a "yes"-"no" judgment about the previous presentation of the word, using the " $d$ " and " $k$ " keys of the keyboard. If necessary, responses were keyed in by the researcher. Once finished, participants were asked to explain in writing what they had based their responses on during the task. None of the participants admitted to basing their responses on any particular feature of the letters. The $\mathrm{O}$ condition was identical to the NO condition, except for stimulus composition, as described previously.

\section{Statistical Analyses}

Hits, false alarms, and discrimination and criterion indices ${ }^{1}$ were analyzed by means of mixed analyses of variance. Although the analyses of the three dependent variables were of principal interest, analyses of hits and false alarms were also performed to correctly interpret the changes in discrimination, as this was the only variable free from criterion biases. For the calculation of the discrimination indices, all proportions were corrected by adding 0.5 to each frequency and dividing the total by the number of trials plus one (Snodgrass \& Corwin, 1988), thereby avoiding undefined $d^{\prime}$ values at proportions of 0 and 1 . The analyses of both hits and discrimination and criterion indices passed the Box' test of equality of covariance matrices. However, the false alarm dependent variable did not pass the test. We decided to perform a square root transformation of all false alarm proportions to correct this deviation. The transformed variable produced a nonsignificant test. In addition, we performed a Levene's test for the equality of variances of the between subject variable for each level of the within factor. The results indicated that variances of the two levels of the within subject factor were homogeneous. Only when the Levene's test was performed for discrimination variances on the $\mathrm{O}$ condition was a significant result obtained, $F(5,92)=2.43, p=$ .041. Given that the ANOVA is very robust to small departures of normality, and that this effect was produced in the $\mathrm{O}$ condition, we decided not to use alternative dependent measures that were less potent. However, as expected, the analysis of hits minus false alarms as an alternative dependent variable produced the same results. For the ANOVAs, the effect sizes reflected the $\eta^{2}$ values as calculated by SPSS and a Bonferroni adjustment was carried out for multiple comparisons. SPSS version 15.0 was used for the analyses and $G^{*}$ Power 3 for the power and sensitivity analyses (Faul, Erdfelder, Lang, \& Buchner, 2007) when necessary.

\section{Results}

The neuropsychological data (see Table 1) show a pattern of deficits coherent with the nature of the different groups. In particular, the GDS (Reisberg, 1982) and the MMSE (Folstin et al., 1975) showed that the control subjects and the two advanced Parkinson groups without dementia were at the same stage, although the advanced Parkinson group approached the scale level of the two dementia groups. The PDD and DLB groups showed visuospatial and visuo-constructive deficits, in addition to lexical access and naming deficits. PDD and DLB patients showed deficits in working and episodic memory. The pattern of neurosychological deficits were identical between DLB and PDD patients in most tests, and were in accordance with those of other published reports (e.g., Mondon et al., 2007). Symptom severity, as assessed by the Hoehn and Yahr (1967) scale, reflects the groups in this study. Table 2 presents the average raw data obtained by condition (O vs. NO) and discrimination indices $\left(d^{\prime}\right)$ and response criteria (C).

A mixed analysis of variance of six groups by two conditions (NO vs. O) showed the effect of condition on hits to be significant, $F(1,92)=15.24, \mathrm{MSe}=0.01, \eta_{\mathrm{p}}^{2}=0.14 p<.01$, indicating that performance in the NO condition was superior to that in the $\mathrm{O}$ condition. Group was marginally significant, $F(5,92)=2.18$, $\eta_{\mathrm{p}}^{2}=0.11, \mathrm{MSe}=0.04, p=.063$, but the interaction was not significant $F(5,92)<1$. The PD with dementia group gave the worst performance in overall hits (0.63), followed by the DLB group (0.68).

As indicated in the statistical analysis section, the proportions of false alarms were submitted to a square root transformation to achieve normality. The NO condition produced less false alarms, $F(1,92)=20.41, \mathrm{MSe}=0.019, \eta_{\mathrm{p}}^{2}=0.18, p<.001$. False alarms differed marginally among the groups, $F(5,92)=12.19$, MSe $=0.06, \eta_{\mathrm{p}}^{2}=0.40, p<.001$, and interaction proved to be significant, $F(5,92)=3.12, \mathrm{MSe}=0.019, p<.05$. The analysis of the interaction indicated that the young, healthy controls and PD1 group produced less false alarms in the NO than in the $\mathrm{O}$ condition. The DLB group had a marginally significant lower

\footnotetext{
${ }^{1}$ Discrimination, $d^{\prime}=\mathrm{z}($ Hits $)-\mathrm{z}($ False Alarms $)$; criterion, $\mathrm{c}=-0.5$ $[z$ (Hits) $+\mathrm{z}$ (False Alarms) $]$, where $\mathrm{z}$ is the standard score for each proportion (Macmillan \& Creelman, 1991).
} 
Table 2

Means (and SE) of Hits (H), False Alarms (FA), Discrimination (d') and Criterion (C) According to Group (Healthy Young Controls, Y; Elderly Healthy Controls, CO; Early Parkinson's, PD1; Advanced Parkinson's, PD2; Parkinson's With Dementia, PDD; and Dementia With Lew Bodies, DLB) and Condition

\begin{tabular}{|c|c|c|c|c|c|c|c|c|}
\hline \multirow[b]{2}{*}{ Group } & \multicolumn{4}{|c|}{ Nonoverlapping } & \multicolumn{4}{|c|}{ Overlapping } \\
\hline & $\mathrm{H}$ & FA & $d^{\prime}$ & $\mathrm{c}$ & $\mathrm{H}$ & FA & $d^{\prime}$ & $\mathrm{c}$ \\
\hline $\mathrm{Y}$ & $0.81(.04)$ & $0.08(.04)$ & $2.45(.20)$ & $0.35(.10)$ & $0.71(.05)$ & $0.18(.04)$ & $1.59(.16)$ & $0.19(.12)$ \\
\hline $\mathrm{CO}$ & $0.77(.04)$ & $0.11(.04)$ & $2.28(.20)$ & $0.27(.11)$ & $0.68(.05)$ & $0.16(.04)$ & $1.58(.16)$ & $0.32(.12)$ \\
\hline PD1 & $0.81(.03)$ & $0.13(.04)$ & $2.27(.18)$ & $0.18(.10)$ & $0.72(.04)$ & $0.23(.04)$ & $1.44(.14)$ & $0.10(.11)$ \\
\hline PD2 & $0.69(.03)$ & $0.12(.04)$ & $1.72(.18)$ & $0.34(.10)$ & $0.62(.04)$ & $0.18(.04)$ & $1.37(.15)$ & $0.34(.11)$ \\
\hline PDD & $0.63(.05)$ & $0.51(.05)$ & $0.36(.25)$ & $-0.16(.14)$ & $0.63(.06)$ & $0.44(.06)$ & $0.51(.20)$ & $-0.11(.15)$ \\
\hline DLB & $0.71(.04)$ & $0.38(.04)$ & $1.02(.19)$ & $-0.15(.11)$ & $0.66(.04)$ & $0.44(.05)$ & $0.66(.16)$ & $-0.13(.12)$ \\
\hline
\end{tabular}

number of false alarms in NO than in $\mathrm{O}$, and no difference was detected for the PDD group $t(15)=5.03, p<.001, t(15)=2.73$, $p<.01, t(19)=3.95, p<.001, t(18)=1.39, p=.09, t(9)=0.54$, $p=.29$, respectively. The individual analyses of hits and false alarms indicated that, independently of group, participants used familiarity to increase their hit and decrease the false alarm rates, and that performance was improved via both changes.

With regards to $d^{\prime}$, the analysis showed that NO led to better performance than the $\mathrm{O}$ condition, $F(1,92)=36.40$, MSe $=0.31$, $\eta_{\mathrm{p}}^{2}=0.28, p<.01$, and that the PDD and DLB groups gave a poorer performance than the remaining groups, $F(5,92)=15.69$, MSe $=0.73, \eta_{\mathrm{p}}^{2}=0.46, p<.01$ and Tukey's tests. It was also confirmed that all groups differed from zero. ${ }^{2}$ More importantly, the interaction of condition and group was also significant, $F(5$, $92)=3.13, \mathrm{MSe}=0.31, \eta_{\mathrm{p}}^{2}=0.15, p<.05$. To analyze this significant interaction, six post hoc $t$ tests were calculated to compare the $d^{\prime}$ s of the NO versus $\mathrm{O}$ condition across groups. This contrast reflected the familiarity effect, and indicated that there were significant differences in the Y, CO, PD1, and DLB groups, $t(15)=4.73, t(15)=4.04, t(19)=3.63, p<.01$, and $t(16)=2.15, p<.05$, respectively, that there was no difference in the PDD group, $t(9)<1$, and that the difference was only marginally significant in the PD2 group, $t(18)=1.83, p=.08$. This is the most important result in relation to the evaluation of familiarity, as it indicates that perceptual familiarity was used independently of considerably varying performance levels and showed no deficits in the young and control samples or PD1 and DLB groups, and only marginally so in the case the PD2 group. However, the PDD group was unable to use perceptual familiarity in its responses. Although the PDD sample was smaller than the other patient groups and the sensitivity of the $t$ test was lower (only large effects were detectable in this case), a trend toward a deficit in familiarity was present in the PD2 sample, in accordance with the published evidence reviewed in the introduction (see Whittington et al., 2000). Considering these factors together, a clear deficit would appear to exist in Parkinson's patients with dementia.

The significant interaction of group and condition was caused by the deficit in familiarity of the PDD group. However, as stated in the introduction, we expected that the Parkinson's group without dementia would not show any deficit in recognition tasks because of the low retrieval requirement of the task. In this case, the interaction between group (excluding the two dementia Groups DLB and PDD) and condition should have been nonsignificant, which was in fact the case in the present study, $F(3,67)=1.48$,
MSe $=0.34, n s$. According to past data published by our group (Algarabel et al., 2009; Algarabel et al., 2010), the familiarity effect expected with Spanish materials could be considered of a medium size (see Cohen, 1988). However, even assuming a power of 0.80 , an alpha of 0.05 and $N=71$ participants, a small effect should have been detected ( $f=0,20$ ). Moreover, assuming a small effect and alpha of $0.05 \mathrm{a}$, a post hoc power analysis of this key interaction with $N=71$ gave a value of 0.79 . Based on this combined power-sensitivity analysis, we can conclude that our experimental design is able to detect a small effect and is, therefore, considerably powerful, which gives credibility to the null effect observed. This analysis produced even better results when the DLB were included; although a dementia group, its final familiarity performance was similar to that of controls and Parkinson groups, with the exception of PDD.

Finally, the analysis of the criterion data, reflecting the willingness to say "yes" by the participants, indicated that there were significant differences among groups, $F(5,92)=4.14, \mathrm{MSe}=.34$, $\eta_{\mathrm{p}}^{2}=0.18, p<.01$. A post hoc Tukey's test showed that the differences between PD2-DLB, PD2-PDD, and DLB-CO were significant. Contrasting these six group criteria means from zero by means of one-sample $t$ tests revealed that only the means of the $\mathrm{PD} 2, \mathrm{CO}$, and Y groups differed from zero, $t(18)=3.96, p<.01$, $t(15)=2.70, p<.05$, and $t(15)=3.09, p<.01$, respectively. When negative, this dependent variable indicates the willingness to indicate that a word has been seen (liberal response).

\section{Discussion}

The purpose of the present study was to assess familiarity recognition in patients with Parkinson's disease with and without dementia, and with dementia caused by Lewy Bodies. The results confirm that there was no familiarity deficit in early stage PD patients without dementia or DLB patients. On the other hand, the familiarity effect in late stage PD patients was marginally significant, and was completely absent in PD patients with dementia. Importantly, the performances of DLB and PD with dementia patients were similar, and became severely undermined when perceptual familiarity was not used. According to the logic set out in the introduction, we attribute the overall deficits in recognition

\footnotetext{
${ }^{2}$ PD1: $t(19)=12.35$; PD2: $t(18)=12.01$; DLB: $t(16)=6.76$; PDD: $t(9)=5.13$; CO: $t(15)=10.21 ; \mathrm{Y}: t(15)=9.81$, all $p \mathrm{~s}<.001$.
} 
in the DLB group to deficits in recollection and other nonretrieval related factors, given that their familiarity abilities seemed to be well preserved.

In this discussion, we would like to point out some aspects of the recognition task used in this study. Second, we would like to discuss the merit of the retrieval deficit hypothesis. Third, we compare Parkinson with dementia patients to DLB patients, and discuss the differences between the deficits shown by these two groups and those of mild cognitive impaired and Alzheimer patients.

This is the only occasion we know of in which familiarity recognition in Parkinson's disease has been assessed directly. A previous report by Davidson et al. (2006) used indirect approaches, evaluating changes in the word frequency mirror effect and subjective remember-know judgments, and using the process dissociation procedure to estimate recollection and familiarity. However, its results are in considerable contrast with other evidence found in the literature. In our study, the direct fluency produced by the repeated presentation of letters permitted the possibility of its use at retrieval. The introduction of an additional "healthy" young participant group allowed us to evaluate the retrieval requirement of the task. The fact that performance in the overall yes-no test and in the familiarity estimation were similar in young and old healthy controls indicates that the retrieval burden introduced by the task was low. This interpretation is reinforced by the small differences in hits between controls and the PDD group. The experimental literature (e.g., Old \& Naveh-Benjamin, 2008; Zacks \& Hasher, 2006) indicates that when a memory task relies heavily on recollection, young participants perform better.

Can the deficits in familiarity observed in this research be attributed to lapses of attention at encoding? This seems unlikely. First, perceptual familiarity as manipulated in this study is automatic, and in consequence does not require conscious attention. Participants were unaware of the experimental manipulation, but despite this unconscious effect, they used it at retrieval. Second, patients with Lewy Body dementia are characterized by severe attention deficits, but despite this disadvantage they performed "normally" in the NO condition. Therefore, we attribute the effect to a retrieval rather than an encoding effect.

We believe that the results of our study is consistent with most relevant data previously published on this topic and clarify some of the apparent inconsistencies that have plagued this area of research with regard to the retrieval deficit hypothesis of memory deficits in Parkinson's disease. A key point of this conclusion is the separate consideration of recollection and familiarity as dual cognitive processes involved in recognition performance. Our view is that inconsistencies in the observation of recognition deficits in PD are related to the balance between recollection and familiarity required by the implementation of a particular recognition task. The more recollection a task requires, the more probable it is that a deficit will be observed. On the contrary, the more a task involves familiarity, the lower is the probability that a deficit will be observed. Based on this analysis and our data, we endorse the retrieval deficit hypothesis (see Higginson et al., 2005), which establishes that PD patients may encode information but find increasing difficulties in responding to tasks with high retrieval requirements. The separate considerations of recollection and familiarity within recognition may help to explain the contradiction between our findings and those obtained by Higginson et al.
(2005). The latter authors applied the California Verbal Learning Test to measure recognition and free and cue recall in advanced stage PD patients with and without dementia and compared their performance with the normative data. The low performance in recognition and cue recall of PD patients and even lower performance of those with dementia led the authors to conclude that the evidence challenged the retrieval deficit hypothesis. As discussed in the introduction, we believe that it is misleading to contrast data across tasks with simplistic assumptions, a view that is supported by the traditional generation-recognition hypothesis, which attributed difference across tasks to the extra step of generating a mental candidate for a response in free recall and not in recognition (e.g., Crowder, 1976). The generation-recognition hypothesis is not supported by current empirical evidence (see Crowder, 1976, for old data). In fact, Higginson et al. reported the presence of a prefrontal dysfunction in their PD patients. They interpreted this as an explanation for the deficits found, but we interpret it as an indication of a deficit in recollection, and, therefore, a plausible explanation for why deficits are observed in recognition tasks with PD patients. It is of interest that, in their study and in ours, the fall in performance attributable to recollection was because of a higher level of false alarms and despite a lack of shift in criterion. Some researchers have attributed this phenomenon to an overreliance on familiarity in the face of recollection deficits (Budson, Todman, \& Schacter, 2006). Data obtained with implicit memory tasks reinforce the conclusion that familiarity is preserved or deteriorates to a lower extent than recollective processes in Parkinson's patients. When tested in implicit tasks with verbal stimuli, PD patients without dementia appear to show no memory deficits, although they do when dementia is associated with Parkinson and verbal priming is measured (Gawrys et al., 2008; Siegert et al., 2006).

For very different reasons, we believe that our results contradict those of Davidson et al. (2006) and provide a more coherent picture of the performance of PD patients in terms of recognition memory. According to Davidson et al. (2006), the deficits in recognition memory, sometimes unreliably observed, are due mainly to deficits in familiarity. They go so far as to conclude that recollection is either intact or almost preserved in PD patients. If this were the case, it would be impossible to comprehend the deficits observed in PD patients when responding to free-recall tasks, a fact beyond any doubt (e.g., Emre, 2003). How could it be that Parkinson's patients are less capable of strategically retrieving past information and are at the same time as efficient as controls, or even more so, in the estimation of the same component through recognition? We believe that the type of task and estimation procedures used to estimate recollection and familiarity lie behind this incongruence.

More importantly, what does familiarity and implicit memory evaluation by clinical entities tell us about these parameters? First, dementias that begin in medial areas, such as Alzheimer's disease, produce deficits in recall and recognition. On the other hand, neurodegenerative disorders such as Parkinson's disease without dementia are related with deficits in recall but a lack of or small deficits in recognition (Whittington et al., 2000), indicating that the involvement of these deficits, whose origin is in the temporal lobe, occur at a late stage and are a consequence of the extension of the illness to higher cortical structures. Parkinson's disease with dementia and DLB produce deficits in both tasks, although these deficits seem to be more pronounced in the latter group (Mondon 
et al., 2007). Finally, as occurs in Parkinson's sufferers, Huntington's patients also exhibit lower deficits in recognition than in recall (Montoya, Pelletier, Menear, Duplessis, Richer, \& Lepage, 2006), although there is extensive debate regarding the status of these patients' recognition memory deficits. Moreover, direct assessment of familiarity deficits in mild cognitive impairment, Alzheimer's disease (Algarabel et al., 2009; Wolk, Signoff, \& DeKosky, 2008) and Parkinson's disease with dementia (present study) indicates that the use of familiarity is severely undermined in these conditions, but is normal in DLB (present study). A recent comparison of the memory profiles of DLB and AD patients (Hamilton et al., 2004) indicated, in line with our argument, that the former group yields better savings scores in recognition than the latter group. In general, DLB patients show less severe episodic memory deficits than AD patients, despite suffering similar levels of dementia. This is consistent with previous studies (e.g., Barber, McKeith, Ballard, Gholkar, \& O'Brian, 2001) indicating that the medial temporal structures involved in memory are more severely affected in AD than in DLB. The literature on implicit memory indicates that the observed pattern of results (Heindel et al., 1989) also differentiates patients with subcortical and cortical dementias. Considered as a whole, this pattern of data provides a consistent differentiation between subcortical and cortical dementias, although disease severity is characterized by a generalized involvement of higher cortical structures in both. Moreover, the present study provides evidence that the origin of dementia in Parkinson's patients may be heterogeneous, and could originate from coincident AD-pathology or Lewy-Body degeneration in different structures. In the past (e.g., Emre, 2003), it has been noted that a proportion of patients with PD with dementia exhibit a similar pathology to those with $\mathrm{AD}$ dementia, and that this similarity becomes more pronounced as the disease progresses. Given the differentiation made in this study between DLB and PDD patients, our data endorse this observation. Nonetheless, it must be stressed that the knowledge possessed at this point in time is not sufficient to offer a completely coherent parallelism between neural substrates of recollection and familiarity and the evolution of Parkinson's and related disorders. Whereas most neuroscientists and experimental psychologists attribute the first manifestations of deficits in recognition to neurodegenerative processes that take place in the temporal lobe, anatomical data (Braak, Rub, \& del Tredici, 2003) indicate that the first clinical manifestations of the illness occur when the pathology reaches the anteromedial temporal mesocortex. "Behavioral" data indicate that there is no deficit in recognition at this stage, which is in accordance with the present results. As discussed previously, it is too early to establish an exact parallelism between neurodegenerative progression and recognition performance. There are many other structures involved in recognition memory (temporal-parietal-prefrontal involvement) to discard the initial anatomical hypothesis used as the starting point of our research.

We must acknowledge the limitations of our study. First, we have attempted to empirically define familiarity in the most direct way possible to avoid assumptions that are sometimes difficult to justify. In doing so, we have defined familiarity in a perceptual and phonological dimension. It may be necessary to generalize and apply the present results to a similar paradigm in which semantic familiarity is involved. Second, the deficits in recollection in our PD patients have been assumed indirectly; that is, considering that recognition is achieved by a combination of familiarity and/or recollection, and given that we detected no deficit in familiarity, we assumed that the global decrease in recognition observed in the patients was because of deficits in recollection. Proof of this assumption must be provided by further experiments. Finally, we believe that recognition performance in patient populations would greatly benefit from event-related potentials or/and neuroimaging experiments that may provide more direct evidence to endorse the premise outlined in the introduction and which has motivated the present work.

\section{References}

Aarsland, D., Brannick, K., Larsen, J. P., Tysnes, O. B., \& Alves, G. (2008). Cognitive Impairment in incident, untreated Parkinson disease: The Norwegian ParkWest study. Neurology, 72, 1121-1126.

Alameda, J. R., \& Cuetos, F. (1995). Diccionario de frecuencias de las unidades lingü, sticas del castellano [Frequency dictionary of the linguistic units of spanish]. Oviedo: Universidad de Oviedo, Departamento de Psicología.

Algarabel, S., Pitarque, A., Tomás, J. M., \& Mazón, J. F. (2010). Explorations on familiarity produced by words with specific combinations of letters. The European Journal of Cognitive Psychology, 22, 265-285.

Algarabel, S., Escudero, J., Mazón, J., Pitarque, A., Peset, V., \& Lacruz, L. (2009). Familiarity-based recognition in the young, healthy elderly, mild cognitive impaired and Alzheimer's patients. Neuropsychologia, 47, 2056-2064.

American Psychiatric Association. (2000). Diagnostic and statistical manual of mental disorders (4th ed., text revision). Washington, DC: Author.

Barber, R., McKeith, I. G., Ballard, C., Gholkar, A., \& O’Brian, J. T. (2001). A comparison of medial and lateral temporal lobe atrophy in dementia with Lewy Bodies and Alzheimer's disease: Magnetic resonance imaging volumetric study. Dementia \& Geriatric Cognitive Disorders, 12, 198-205.

Braak, H., Rub, U., Gai, W. P., \& Del Tredici, K. (2003). Idiophatic Parkinson's disease: Possible routes by which vulnerable neuronal types may be subject to neuroinvasion by an unknown pathogen. Journal of Neural Transmission, 110, 517-536.

Budson, A. E., Todman, R. W., \& Schacter, D. L. (2006). Gist memory in Alzheimer s disease: Evidence from categorized pictures. Neuropsychology, 20, 113-122.

Cohen, J. (1988). Statistical power analysis for the behavioral sciences (2nd ed.). Hillsdale, NJ: Erlbaum.

Costa, A., Peppe, A., Caltagirone, C., \& Carlesimo, G. A. (2008). Prospective memory impairment in individuals with Parkinson's disease. Neuropsychology, 22, 283-292.

Crowder, R. G. (1976). Principles of learning and memory. Hillsdale, NJ: Erlbaum.

Cummings, J. L., \& Benson, D. F. (1984). Subcortical dementia: A review of an emerging concept. Archives of Neurology, 41, 874-879.

Davidson, P., Anaki, D., Saint-Cyr, J. A., Chow, T., \& Moscovitch, M. (2006). Exploring the recognition memory deficit in Parkinson's disease: Estimates of recollection versus familiarity. Brain, 129, 1768-1779.

Emre, M. (2003). Dementia associated with Parkinson's disease. The Lancet Neurology, 2, 229-237.

Faul, F., Erdfelder, E., Lang, A.-G., \& Buchner, A. (2007). G*Power 3: A flexible statistical power analysis program for the social, behavioral, and biomedical sciences. Behavior Research Methods, 39, 175-191.

Folstein, M., Folstein, S., \& McHugh, P. (1975). "Mini-Mental State": A practical method for grading the cognitive state of patients for the clinician. Journal of Psychiatric Research, 12, 189-198.

Gawrys, L., Jamrozik, Z., Janik, P., Friedman, A., \& Kaczmarek, L. (2008). Nonverbal deficits in explicit and implicit memory of Parkinson's disease. Acta Neurobiologica Experimentalis, 68, 58-72. 
Hamilton, J. M., Salmon, D. P., Galasko, D., Delis, D. C., Hansen, L. A., Masliah, E., ... Thal, L. J. (2004). A comparison of episodic memory deficits in neuropathologically-confirmed Dementia with Lewy bodies and Alzheimer's disease. Journal of the International Neuropsychological Society, 10, 689-697.

Heindel, W. C., Salmon, D. P., Shults, C. W., Walick, P. A., \& Butters, N. (1989). Neuropsychological evidence for multiple implicit memory systems: A Comparison of Alzheimer's, Huntington's, and Parkinson's disease patients. The Journal of Neuroscience, 9, 582-587.

Higginson, C. I., Wheelock, V. L., Carroll, K. E., \& Sigvardt, K. A. (2005). Recognition memory in Parkinson's disease with and without dementia: Evidence inconsistent with the retrieval deficit hypothesis. Journal of Clinical and Experimental Neuropsychology, 27, 516-528.

Hoehn, M. M., \& Yahr, M. D. (1967). Parkinsonism: Onset, progression, and mortality. Neurology, 17, 427-442.

Hughes, A. J., Daniel, S. E., Kilford, L., \& Lees, A. J. (1992). Accuracy of clinical diagnosis of idiophatic Parkinson's disease: A clinicopathological study of 100 cases. Journal of Neurology, Neurosurgery and Psychiatry, 55, 181-184.

Kaplan, E. F., Goodglass, H., \& Weintraub, S. (1983). Boston Naming Test. Philadelphia: Lea \& Febiger.

Macmillan, N. A., \& Creelman, C. D. (1991). Detection theory: A user's guide. New York: Cambridge University Press.

McKeith, I. (2007). Dementia with Lewy bodies and Parkinson's disease with dementia: Where two worlds collide. Practical Neurology, 7, 374-382. doi: 10.1136/jnnp.2007.134163

McKeith, I. G., Mintzer, J., Aarsland, D., Burn, D., Chiu, H., CohenMansfield, J., ... Playfer, J. (2004). Dementia with Lewy bodies. The Lancet Neurology, 3, 19-28.

McKeith, I. G., Dickson, D. W., Lowe, J., Emre, M., O’Brien, J. T., Feldman, H., ... Yamada, M. (2005). Diagnosis and management of dementia with Lewy bodies: Third report of the DLB Consortium. Neurology, 65, 1863-1872.

Mondon, K., Gochard, A., Marqué, A., Amand, A., Beauchamp, D., Prunier, C., ... Hommet, C. (2007). Visual recognition memory differentiates dementia with Lewy Bodies and Parkinson's disease dementia. Journal of Neurology, Neurosurgery and Psychiatry, 78, 738-741.

Montoya, A., Pelletier, M., Menear, M., Duplessis, E., Richer, F., \& Lepage, M. (2006). Episodic memory impairment in Huntington's disease: A meta-analysis. Neuropsychologia, 44, 1984-1994.

Nissen, M. J., \& Bullemer, P. (1987). Attention requirements of learning: Evidence from performance measures. Cognitive Psychology, 19, 1-32.

Old, S. R., \& Naveh-Benjamin, M. (2008). Differential effects of age on item and associative measures of memory: A meta-analysis. Psychology and Aging, 23, 104-118.

Parkin, A., Ward, J., Squires, E., Furbear, H., Clark, A., \& Townshend, J. (2001). Data-driven recognition memory: A new technique and some data on age differences. Psychonomic Bulletin and Review, 8, 812-819.

Peigneux, P., Meulemans, T., Van der Linden, M., Salmon, E., \& Petit, H. (1999). Exploration of implicit artificial grammar learning in Parkinson's disease. Acta Neurologica Belgica, 99, 107-117.

Peña-Casanova, J., Gramunt-Fombuena, N., \& Gich-Fulla, J. (2004). Tests neuropsicológicos. Fundamentos para una neuropsicología clínica basada en evidencias [Neuropsychological Tests. Foundations for a clinical neuropsychology based on evidences]. Barcelona: Masson.
Reber, P. J., \& Squire, L. R. (1999). Intact learning of artificial grammars and intact category learning by patients with Parkinson's disease. Behavioral Neuroscience, 113, 235-242.

Reisberg, B., Ferris, S. H., de Leon, M. J., \& Crook, T. (1982). The Global Deterioration Scale for assessment of primary degenerative dementia. American Journal of Psychiatry, 139, 1136-1139.

Reitan, R. M. (1958). Validity of the Trail Making test as an indicator of organic brain damage. Perceptual \& Motor Skills, 8, 271-276.

Rey, A. (1941). L'examen psychologique dans les cas d'encephalopathie traumatique [Psychological examination of cases of traumatic encephalopathy]. Archives de Psychologie, 28, 286-340.

Schneider, W., Eschman, A., \& Zuccolotto, A. (2002). E-Prime reference guide. Pittsburg: Psychology Software Tools Inc.

Sheikh, J. L., \& Yesavage, J. A. (1986). Geriatric Depression Scale (GDS). Recent evidence and development of a shorter version (Spanish version by Martínez de la Iglesia, Onís, Dueñas, Albert, Aguado, Luque, 2002). Clinical Gerontology, 5, 165-172.

Siegert, R. J., Taylor, K. D., Weatherall, \& Abernethy, D. A. (2006). Is implicit sequence learning impaired in Parkinson s disease? A metaanalysis. Neuropsychology, 20, 490-495.

Smith, J. G., \& McDowall, J. (2006). When artificial grammar acquisition in Parkinson's disease is impaired: The case of learning via trial-by-trial feedback. Brain Research, 1067, 216-228.

Snodgrass, J. G., \& Corwin, J. (1988). Pragmatics of measuring recognition memory. Journal of Experimental Psychology: General, 117, 34.

Taylor, A. E., Saint-Cyr, J. A., \& Lang, A. E. (1986). Frontal lobe dysfunction in Parkinson's disease. Brain, 109, 845-883.

Toulouse, E., \& Pieron, H. (1986). Toulouse-Piéron (Prueba Perceptiva y de atención). Madrid: Tea Ediciones S. A.

Verbaan, D., Marinus, J., Visser, van Rooden, S. M., Stiggelbout, A. M., Middelkoop, H. A. M., \& van Hilten, J. J. (2007). Cognitive impairment in Parkinson's disease. Journal of Neurology, Neurosurgery, \& Psychiatry, 78, 1182-1187.

Wechsler, D. (1987). The Wechsler Memory scale-Revised. San Antonio, TX: The Psychological Corporation.

Whittington, C., Podd, J., \& Kan, M. (2000). Recognition Memory Impairment in Parkinson's Disease: Power and Meta-Analyses. Neuropsychology, 14, 233-246.

Whittington, C., Podd, J., \& Stewart-Williams, S. (2006). Memory deficits in Parkinson's disease. Journal of clinical and Experimental Neuropsychology, 28, 738-754.

Witt, K., Nuhsman, A., \& Deuschl, G. (2002). Intact artificial grammar learning in patients with cerebellar degeneration and advanced Parkinson's disease. Neuropsychologia, 40, 1534-1540.

Wolk, D. A., Signoff, E. D., \& DeKosky, S. T. (2008). Recollection and familiarity in amnestic mild cognitive impairment: A global decline in recognition memory. Neuropsychologia, 46, 1965-1978.

Yonelinas, A. P. (2002). The nature of recollection and familiarity: A review of 30 years of research. Journal of Memory and Language, 46, 441-517.

Zacks, R. T., \& Hasher, L. (2006). Aging and long-term memory: Deficits are not inevitable. In E. Bialystok \& F. I. M. Craik (Eds.), Lifespan cognition: Mechanisms of change. New York: Oxford University Press.

Received September 21, 2009

Revision received January 25, 2010

Accepted January 25, 2010 